\title{
Factors Responsible for Lower Back Pain in Kendo Practitioners
}

\author{
SHINYA KISHI, RPT, MS ${ }^{1,2)}$, IKUHIRO MORIKITA, MD, PhD ${ }^{1)}$, KYOSUKE TAKASAKI, RPT, MA ${ }^{3)}$, \\ TSUYOSHI YAMAGUCHI, RPT ${ }^{4}$, TOSHIAKI SUZUKI, RPT, PhD ${ }^{3)}$ \\ 1) Osaka University of Health and Sport Sciences, Graduate School, Laboratory of Sports Medical \\ Sciences: Asashirodai 1-1, Kumatori-cho Sennan-gun, Osaka 590-0496, Japan. \\ TEL: +81 72-453-8953, FAX: +816-6624-2799, E-mail: shinya-kazu@h8.dion.ne.jp \\ ${ }^{2)}$ Department of Rehabilitation, Sumiya Orthopedic Hospital \\ ${ }^{3)}$ Clinical Physical Therapy Laboratory, Faculty of Health Sciences, Kansai University of \\ Health Sciences \\ 4) Department of Rehabilitation, Kuzuha Hospital
}

\begin{abstract}
Purpose] Lower back pain is a characteristic of Kendo practitioners which is often encountered clinically. The present study was undertaken to analyze the features of the spinal alignment in Kendo practitioners and their relation to lower back pain. [Subjects and Methods] Of the 32 Kendo practitioners who consulted our hospital with a complaint of lower back pain and received physical therapy during the two years and three months from January 2005 to April 2007, On X-ray, the degree of eight kendo practitioners' spinal curvature was measured by Cobb's method. Inclination and rotation were measured at each intervertebral level of the lumbar spine. The angle of inclination at each intervertebral space (L1/2, L2/ $3, \mathrm{~L} 3 / 4$ and L4/5) was measured. In one of the kendo practitioners, the motion of stepping forward to make a frontal thrust was analyzed three-dimensionally. [Results] During the forward-stepping motion, the thoracic spine showed right convex curvature and the lumbar spine showed left convex curvature, with Cobb's angle being $12.4 \pm 2.5$ degrees for the thoracic spine and $13.7 \pm 1.7$ degrees for the lumbar spine. [Conclusions] These results suggest that shear stress at the left intervertebral joint at the L3/4 and L4/5 levels and stress of the facet joint capsule are involved in lower back pain.
\end{abstract}

Key words: Kendo, Spinal Alignment, Back Pain

(This article was submitted Oct. 22, 2008, and was accepted Dec. 22, 2008)

\section{INTRODUCTION}

The sliding walk is one of the basic motions performed by Kendo practitioners. The hip joint serves as the center during this motion. During Kendo, the practitioner puts the right foot forward and keeps the left foot back, regardless of whether or not the right or left hand is the dominant hand of the practitioner. The motion taken by Kendo practitioners when attacking the opposing party with a bamboo sword also involves marked forward movement of the right foot around the hip joint (hip flexion) and backward kicking with the left foot (hip extension). Kendo practitioners repeat cycles of these motions. Because of this feature of Kendo, the lower back pain characteristic of Kendo practitioners is often encountered clinically. The present study was undertaken to analyze the features of the spinal alignment of frontal thrust in Kendo practitioners and their relation to lower back pain. 
Table 1. Features of Kendo participants

\begin{tabular}{lllll}
\hline Sex & Age & Diagnosis & Symptom & Motion causing aggravation of symptoms \\
\hline Male & 17 & L5/S1 disc herniation & Left lower back pain, fatigue of left leg & Stepping forward, sword pulling \\
Male & 18 & L4/5 disc herniation & Left lower back pain, left leg pain & Stepping forward, pushing sword-guard \\
Male & 18 & L4/5 disc herniation & Bilateral lower back pain, bilateral leg pain & Stepping forward, sword pulling \\
Male & 38 & L5/S1 disc herniation & Left lower back pain, left leg pain & Stepping forward, pushing sword-guard \\
Male & 45 & L4/5/S1 disc herniation & Left lower back pain, fatigue of left leg & Stepping forward, sword pulling \\
Male & 16 & Muscular/fascial lower back pain & Left lower back pain & Stepping forward, sword pulling \\
Female & 16 & Muscular/fascial lower back pain & Left lower back pain & Stepping forward, sword pulling \\
Female & 22 & Muscular/fascial lower back pain & Bilateral lower back pain & Stepping forward, sword pulling \\
\hline
\end{tabular}

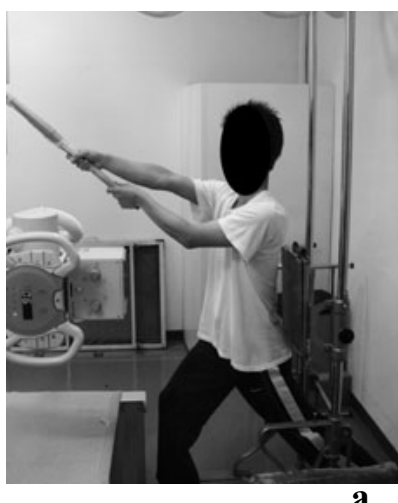

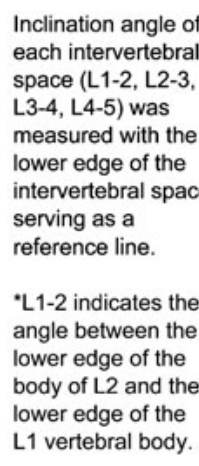
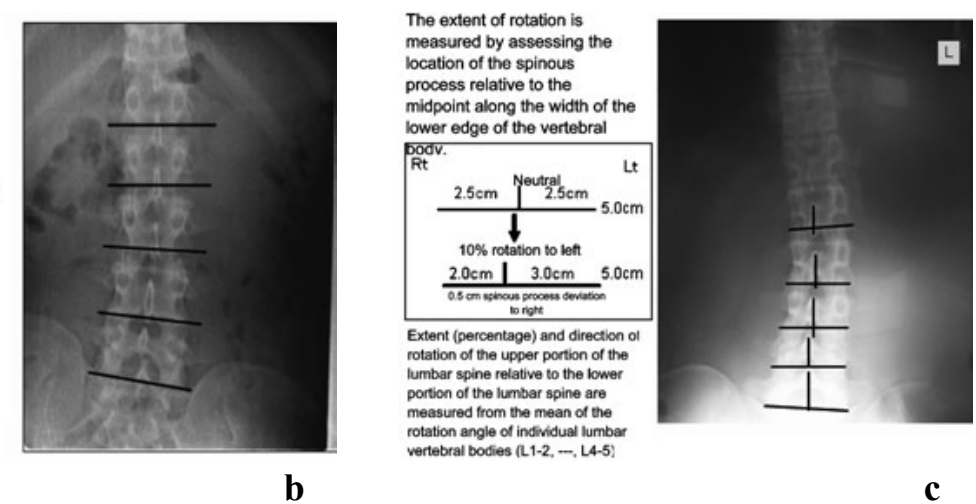

Fig. 1. Radiography of forward-stepping motion (to make a frontal thrust) and analysis of the radiograms.

a: Radiography.

b: Measurement of the angle of inclination for each intervertebral space of the lumbar spine.

c: Measurement of the extent (percentage) of rotation for each intervertebral space of the lumbar spine.

\section{SUBJECTS AND METHODS}

\section{Subjects}

Of the 32 Kendo practitioners who consulted our hospital with a complaint of lower back pain and received physical therapy during the two years and three months from January 2005 to April 2007, eight practitioners who gave consent to this radiography-based study were enrolled. There were six males and two females, with ages ranging from 16 to 45 years $(23.75 \pm 11.27$ years $)$. These practitioners had been practicing Kendo three times or more per week. They had begun Kendo 9-10 years previously. Lumbar disc herniation was diagnosed in five practitioners and muscular or fascial lower back pain in three practitioners (Table 1). The study objective, significance, methods, and privacy protection were explained to the subjects in writing, and each participant provided informed written consent.

\section{Methods}

Frontal x-rays of the entire spinal column and lumbar spine were taken in the standing position and during the forward-stepping motion (Fig. 1a). Alignment was evaluated in the following ways.

On X-rays, the degree of spinal curvature was measured by Cobb's method.

Inclination and rotation were measured at each intervertebral level of the lumbar spine. The angle of inclination at each intervertebral space (L1/2, L2/ 3 , L3/4 and L4/5) was measured, with the lower edge of each intervertebral space serving as the reference line. L1/2 indicates the angle formed by the lower edge of the L2 vertebral body and the lower edge of the L1 vertebral body (Fig. 1b). The extent of rotation at each intervertebral level of the lumbar spine was measured by assessing the location of the spinous process relative to the midpoint of the width of the lower edge of the vertebral body (Fig. 1c). The same examiner measured the radiographic alignment.

Of the eight subjects, one subject (a 38-year-old 


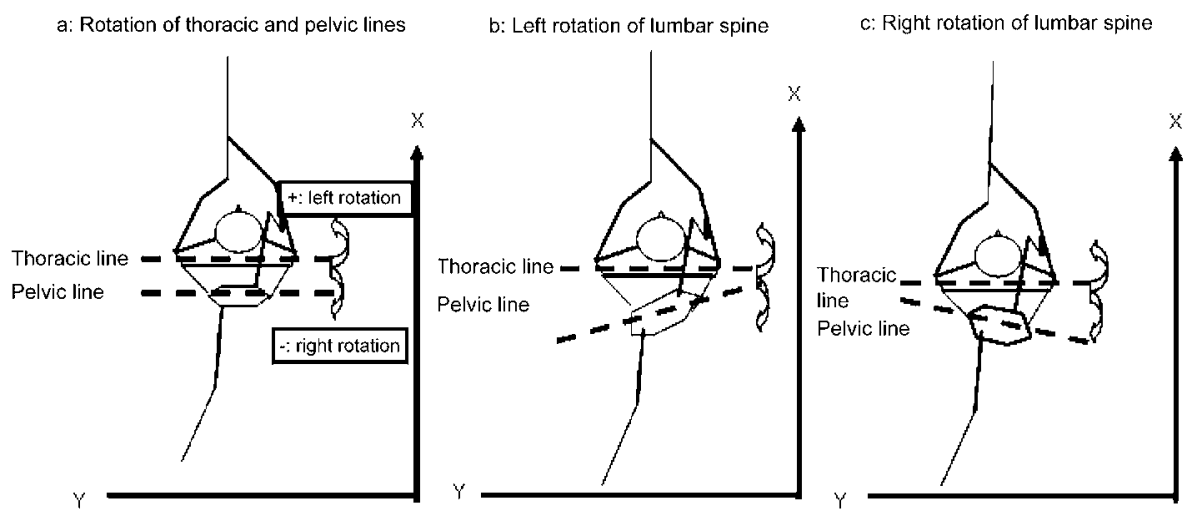

Fig. 2a. Method for measuring thoracic and pelvic rotation and definition of lumbar spine rotation.

a: When viewed from the upper aspect of the body, rotation in a counter-clockwise direction (positive rotation) is expressed as left rotation for both the thoracic and pelvic lines. Rotation in a clockwise direction (negative rotation) is expressed as right rotation for both the thoracic and pelvic lines.

b: A judgment of left lumbar rotation is made if the rotation of the pelvic axis is shifted to the left as compared to the rotation of the thoracic axis.

c: A judgment of right lumbar rotation is made if the rotation of the pelvic axis is shifted to the right as compared to the rotation of the thoracic axis.
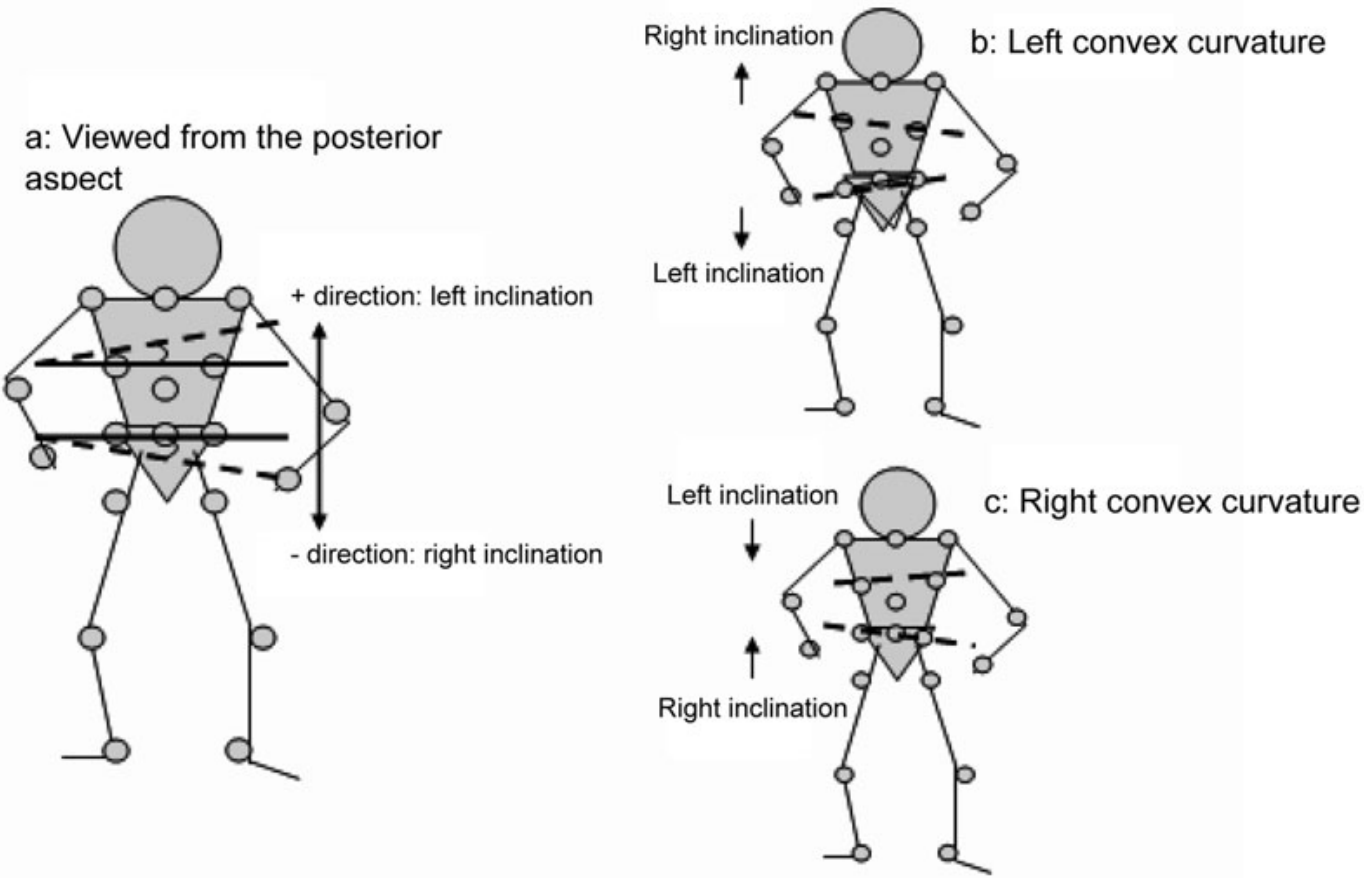

Fig. 2b. Method for measuring thoracic and pelvic inclination and definition of lumbar spine lateral curvature. a: Angle of thoracic and pelvic inclination (Inclination in a counter-clockwise direction (positive inclination) to $\mathrm{Y}$ axis is expressed as left rotation when viewed from the posterior aspect of the body. Inclination in a clockwise direction (negative rotation) is expressed as right inclination.)

$\mathrm{b}$ : A judgment of "left convexity" is made if the pelvic line is shifted to the left as compared to the thoracic line.

c: A judgment of "right convexity" is made if the pelvic line is shifted to the right as compared to the thoracic line. 


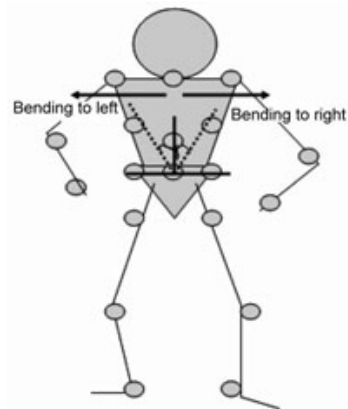

Fig. 2c. Measuring the angle of lateral bending of the lumbar spine.

The angle is rated as 0 degrees if the spinal column line is perpendicular to the pelvic line. A judgment of "bending to the left" is made if the spinal column line seen from the back is bent to left when viewed from the posterior aspect of the body. A judgment of "bending to the right" is made if the spinal column line is bent to the right.

male Kendo participant with a history of lumbar disc herniation) was selected for three-dimensional motion analysis.

The participant stepped forward toward an imaginery opponent, raising the right foot from the floor and dropping the right foot on the floor, to achieve a frontal thrust, as if striking Men after a one-step, one-swing motion. The experimental demonstration was performed only once.

Two units of a compact, simplified motion capture system (UM-CAT II, Unimec Co., Ltd.), capable of three-dimensional coordinate measurement and equipped with two cameras, were placed, one unit in the right anterior area and the other unit in the left anterior area ${ }^{1)}$. Another unit, equipped with two cameras, was set in the right posterior area. In total, six infrared rays cameras were used. Using these cameras, the forwardstepping motion during frontal thrust was captured (imaging rate $180 \mathrm{~Hz}, 30$ frames/second). A lightemitting marker $(2.0 \mathrm{~cm}$ in diameter) was attached to each of 19 sites on the practitione's body. The coordinate of each marker was automatically digitized with the UM-CAT II. From the coordinates thus obtained, three-dimensional coordinates were calculated for a space of $2 \mathrm{~m}$ dimensions along each of the $\mathrm{X}$ axis (direction of advancement), $\mathrm{Y}$ axis (horizontal direction) and $\mathrm{Z}$ axis (vertical direction), using modified DLT (direct linear transformation $)^{2)}$. The difference between the measured value and the value estimated with modified DLT was $0.2 \mathrm{~cm}$ along the $X$ axis, $0.12 \mathrm{~cm}$ along the $\mathrm{Y}$ axis and $0.06 \mathrm{~cm}$ along the $\mathrm{Z}$ axis. The three-dimensional coordinates were subjected to quadratic Butterworth filtering to eliminate high frequency noise, and smoothed with the cut-off frequency set at $6 \mathrm{~Hz}$.

The motion of stepping forward was divided into two phases: the early phase (from lift-of the right foot from the floor to arrival of the right knee at peak height) and the late phase (from arrival of the right knee at peak height to the right foot touching the floor). During each phase, rotation, lateral curvature and lateral bending of the lumbar spine were analyzed .

Lumbar spine rotation: Rotation of the thorax and pelvis was measured by determining the angle formed between the thoracic line (the line joining the lower edge of right and left ribs) or the pelvic line (the line joining the right and left anterior superior iliac spines) and the line perpendicular to the direction of the forward step (Y axis). The rotation angle was defined as follows. The angle was rated as 0 degrees if the thoracic line and the pelvic line were parallel to the direction of advancement when viewed from the upper aspect of the body. Rotation in a counter-clockwise direction (positive rotation) was expressed as left rotation. Rotation in a clockwise direction (negative rotation) was expressed as right rotation. Left lumbar rotation was judged to be present if the rotation of the pelvic axis was shifted to the left as compared to the rotation of the thoracic axis. A judgment of "right lumbar rotation" was made if the rotation of the pelvic axis was shifted to the right as compared to the rotation of the thoracic axis (Fig. 2a).

Lumbar spine lateral curvature: Thoracic inclination and pelvic inclination were measured in the frontal plane by checking the angle formed between the thoracic line (the line joining the lower edge of right and left ribs) or the pelvic line (the line joining the right and left anterior superior iliac spines) and the line perpendicular to the direction of stepping forward (Y axis). The angle of inclination was defined as follows. The angle was rated as 0 degrees if the thoracic line and the pelvic line were parallel to the $\mathrm{Y}$ axis when viewed from the posterior aspect of the body. Inclination in a counter-clockwise direction (positive inclination) was expressed as left rotation. Inclination in a clockwise direction (negative inclination) was expressed as right inclination. A judgment of "left 
Table. 2 Extent (percentage) of rotation for each intervertebral

left rotation of the lower portion of the lumbar spine and a neutral pattern in the upper portion of the lumbar spine (4 cases)

L1 : $0 \%$, L2 : $2.4 \pm 2.6 \%$, L3 : $7.7 \pm 0.5 \%$, L4 : $6.2 \pm 0.5 \%$, L5 : $4.5 \pm 0.6 \%$

$\mathrm{L} 1 / 2: 2.4 \%$ right rotation, $\mathrm{L} 2 / 3: 5.3 \%$ right rotation, $\mathrm{L} 3 / 4: 1.5 \%$ left rotation, $\mathrm{L} 4 / 5: 1.7 \%$ left rotation

left rotation of lumbar spine ( 2 cases)

L1 : $11.0 \%$, L2 $: 10.0 \pm 1.45 \%$, L3 : $5.5 \pm 0.7 \%$, L4 $: 4.0 \%, \quad$ L5 $: 2.5 \pm 0.7 \%$

$\mathrm{L} 1 / 2: 1.0 \%$ left rotation, $\mathrm{L} 2 / 3: 4.5 \%$ left rotation, $\mathrm{L} 3 / 4: 1.5 \%$ left rotation, $\mathrm{L} 4 / 5: 1.5 \%$ left rotation

left rotation of the lower portion of the lumbar spine and right rotation of the upper portion of the lumbar spine ( 2 cases)

L1 : $10.0 \%$, L2 : $13.0 \%$, L3 : $14.5 \pm 0.7 \%$, L4 : $14.0 \%$, L5 : $2.5 \pm 0.7 \%$

$\mathrm{L} 1 / 2: 3.0 \%$ left rotation, $\mathrm{L} 2 / 3: 1.5 \%$ left rotation, $\mathrm{L} 3 / 4: 0.5 \%$ right rotation, $\mathrm{L} 4 / 5: 16.5 \%$ right rotation
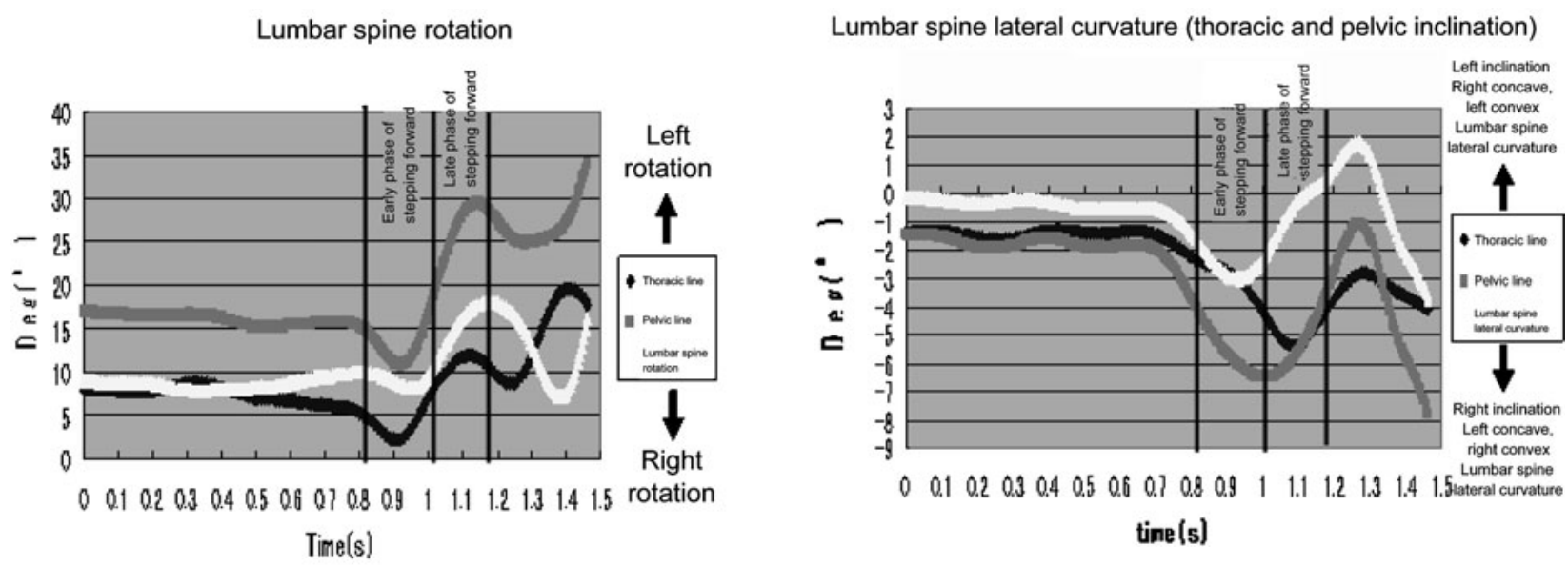

Fig. 3. Lumbar spine motion during stepping forward to make a frontal thrust (analyzed from changes in thoracic and pelvic lines)

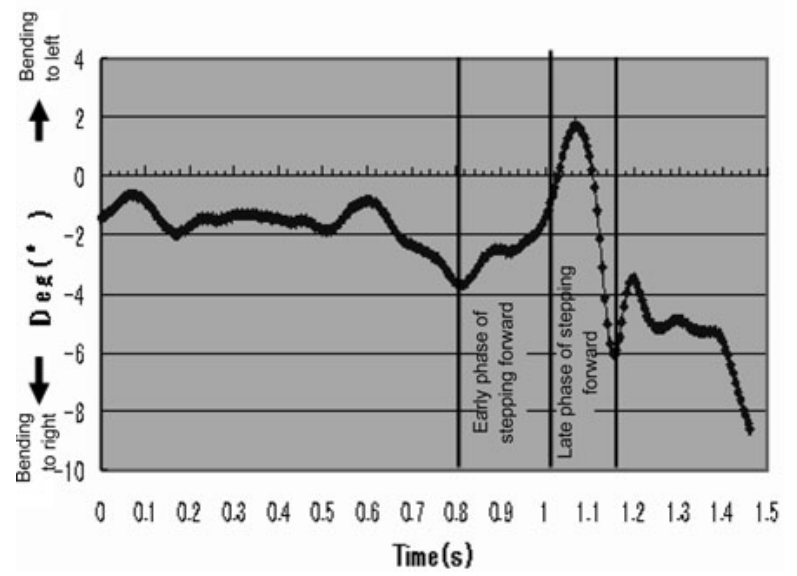

Fig. 4. Lateral bending of lumbar spine during stepping forward to make a frontal thrust 
convexity" was made if pelvic inclination was shifted to the left as compared to the thoracic inclination. A judgment of "right convexity" was made if pelvic inclination was shifted to the right as compared to the thoracic inclination (Fig. 2b).

Lateral bending of the lumbar spine was assessed by measuring the angle formed between the spinal column line (the line joining the spinous processes at L1 and L5 levels) and the pelvic line when viewed from the posterior aspect of the body. Lateral bending of the lumbar spine was defined as follows. The angle was rated as 0 degree if the spinal column line was perpendicular to the pelvic line. A judgment of "bending to the left" was made if the spinal column line was bent to the left. A judgment of "bending to the right" was made if the spinal column line was bent to the right (Fig. 2c).

The relationship between lateral curvature and rotation of the lumbar spine was investigated with Pearson's correlation was applied to data for lateral curvature and rotation of the lumbar spine, using the statistical analysis program SPSS 11.0 for Windows.

\section{RESULTS}

All of the eight Kendo practitioners showed favorable alignment in the standing position. During the forward-stepping motion, the thoracic spine showed right convex curvature and the lumbar spine showed left convex curvature, with Cobb's angle being $12.4 \pm 2.5$ degrees for the thoracic spine and $13.7 \pm 1.7$ degrees for the lumbar spine .

The angle of inclination of the intervertebral space of the lumbar spine was largest at the L3/4 level $(0.25 \pm 0.4$ degrees at $\mathrm{L} 1 / 2,1.25 \pm 1.0$ degrees at $\mathrm{L} 2 / 3,3.37 \pm 1.0$ degrees at $\mathrm{L} 3 / 4$ and $0.88 \pm 0.6$ degrees at L4/5).

In patterns of lumbar spine rotation, four practitioners showed leftward rotation of the lower portion of the lumbar spine and a neutral pattern in the upper portion of the lumbar spine (pattern A). Two practitioners showed leftward rotation of the lumbar spine (pattern B). Two practitioners showed leftward rotation of the lower portion of the lumbar spine and right rotation of the upper portion of the lumbar spine (pattern C). The vertebral body showing the highest degree of rotation was L3 for pattern A (left rotation by $7.7 \pm 0.5 \%$ ), L1 for pattern B (left rotation by $11.0 \%$ ) and L3 for pattern $\mathrm{C}$ (left rotation by $14.5 \pm 0.7 \%$ ). The intervertebral space showing the highest degree of rotation was $\mathrm{L} 2 / 3$ for pattern A (5.3\% right rotation of the upper vertebral body relative to the lower vertebral body), L2/3 for pattern B (4.5\% left rotation of the upper vertebral body relative to the lower vertebral body) and $\mathrm{L} 4 / 5$ for pattern $\mathrm{C}(16.5 \%$ right rotation of the upper vertebral body relative to the lower vertebral body) (Table 2).

During the first half of the early phase of the forward-stepping motion, both the thorax and pelvis showed right rotation. During the second half of the early phase, both showed left rotation. In addition, during the late phase of the forward-stepping motion, the thorax and pelvis showed increased rotation to the left from the first to second half of this phase, but both thorax and pelvis showed right rotation at the end of this phase. The rotation of the lumbar spine during the early phase of the forwardstepping motion was similar to that of the thorax and pelvis, but showed an increase in left rotation during the late phase of this motion (Fig. 3).

Both the thoracic spine and pelvis showed right inclination during the early phase of the forwardstepping motion and left inclination during the late phase of the same motion. However, right inclination of the pelvis was smaller than that of the thorax during the early phase and left inclination of the pelvis was larger during the late phase. As a result, the pelvis remained inclined to the left during this motion and the lumbar spine showed lateral curving (left convex curvature) during both early and late phases of the motion (Fig. 3).

The lumbar spine showed bending to the left during the early phase of the forward-stepping motion and bending to the right during the late phase. The angle of rightward bending was maximal at the instant when the right foot touched the floor (Fig. 4).

(1) As the lumbar spine underwent left convex curving, the lower level of the lumbar spine showed rotation to the left; and (2) as the lumbar spine underwent rightward rotation, the lower level of the lumbar spine showed rightward rotation.

\section{DISCUSSION}

As far as sports injuries seen in Kendo practitioners are concerned, lower back pain is second most frequent after heel pain ${ }^{3)}$.

Although the overall incidence of injury is lower among Kendo practitioners than among other sports 
players $^{4)}$, a high prevalence of lower back pain among those continually practicing Kendo is a characteristic sports injury associated with Kendo ${ }^{5}$. Lower back pain in Kendo practitioners, encountered during routine clinical practice, can be characterized by reproduction or intensification of the pain in the lower left part of the lumbar spine during the forward-stepping motion. In addition, tenderness is often seen on the left side (at L3/4/5/ S1 levels). We are of the opinion that this kind of pain is associated with the forward-stepping motion of Kendo practitioners ${ }^{5-8}$ ). The forward-stepping motion of Kendo involves a large forward swing of the right foot and backward kicking of the left foot from a position where the right hand and foot are forward and the left hand and foot are behind. During this motion, the lumbar spine extends and the lower portion of the lumbar spine rotates and bends left relative to the upper portion of the lumbar spine. The resultant compressive stress on the lumbar facet joint probably causes lower back pain. At the 11 th meeting of the Kansai Society of Clinical Sports Medicine, We reported that lordosis (forward curvature) of the lumbar spine intensified during the forward-stepping motion in Kendo practitioners who had complained of lower back pain $^{9}$. We additionally thought that if the strength of the abdominal and gluteus muscles is reduced and the range of hip motion is narrowed, extension of the lumbar spine during this motion intensifies, leading to higher compressive stress on the facet joint. In the present study, however, radiographic evaluation of alignment during the forwardstepping motion and three-dimensional analysis of the forward thrusting motion revealed bending of the spinal column to the right and left convex curvature of the lumbar spine at the end of the late phase of the forward-stepping motion (the point when the right foot touches the floor, causing the highest impact). This finding suggests that the compressive stress on the left facet joint is lower than that on its counterpart on the right. We therefore believe that lower back pain in Kendo practitioners is attributable to a stretching stress on the capsule of the left facet joint and deep-seated muscles (e.g., multifidus muscle), and that this stress is highest at the L3/4 level, at which level the angle of inclination of the lumbar vertebral body was found to be highest. During clinical practice, we often find that the paravertebral muscle (PVM) at this site is in spasm. The three Kendo practitioners with muscular or fascial lower back pain complained of pain at this site. Left rotation of the lower portion of the lumbar spine, relative to the upper portion of the lumbar spine, accompanied left convex curvature of the lumbar spine, and significant correlation between these changes was revealed by three-dimensional analysis. This rotation of the lower portion of the lumbar spine is attributable to coupling motion ${ }^{10-13)}$. It seems that this rotation of the spine causes shear stress on the lumbar facet joint. When rotation of the lumbar spine was evaluated on radiograms, three patterns of rotation were identified: pattern A (left rotation of the lower portion of the lumbar spine and a neutral pattern in the upper portion of the lumbar spine), pattern B (left rotation of the lumbar spine) and pattern $\mathrm{C}$ (left rotation of the lower portion of the lumbar spine and right rotation of the upper portion of the lumbar spine). In six practitioners showing pattern $\mathrm{A}$ or $\mathrm{B}$, rotation at $\mathrm{L} 2 / 3$ was marked. In two cases showing pattern $\mathrm{C}$, rotation at L 4/5 was marked. These results suggest that the shear stress on the facet joint arising from lumbar spine rotation is highest at the L2/3 and L4/5 levels. However, in clinical cases, pain was not seen at the L2/3 level. Furthermore, in two practitioners showing pattern $\mathrm{C}$, rotation at the L $4 / 5$ level $(16.5 \%)$ was more marked than rotation at the $\mathrm{L} 2 / 3$ level $(4.5-5.3 \%)$. It seems therefore that only pattern $\mathrm{C}$ is associated with lower back pain arising from lumbar spine rotation. Although the angle of lumbar spine rotation was not measured in the present study, Fujii et al. previously analyzed the mean rotation angle during maximum trunk rotation (mean 56.1 degrees on one side) using a new noninvasive three-dimensional motion analysis system based on 3D-MRI, and reported that the range of rotation of each intervertebral space was about 1-2 degrees on one side ${ }^{14)}$. Mimura also reported similar results from three-dimensional measurement of bone arrangement ${ }^{15}$ ). Taken together, these findings suggest that excessive rotation at $\mathrm{L} 4 / 5$ level was present in cases showing pattern C. Because these two practitioners showing pattern $C$ had lumbar disc herniation at the L4/5 level, the following mechanism for onset of lower back pain seems probable: degeneration of the intervertebral disc $\rightarrow$ instability of the facet joint $\rightarrow$ excessive rotation of the facet joint $\rightarrow$ internal disorders such as disc herniation $\rightarrow$ onset of lower back pain. 
In addition, deviation of the axis of motion seen around L4 (at L 3/4 and L4/5 levels) in all practitioners may also be associated with lower back pain. On the basis of these results, we may say that the lower back pain seen in Kendo practitioners, associated with the forward-stepping motion (for a frontal thrust), is attributable to the following factors: (1) stretching stress on the left facet joint capsule and deep-seated muscles on the left side of L3/4 and L4/5 levels following bending of the trunk to the right and (2) shear stress on the facet joint and the L4/5 disc due to coupling motion following bending of the trunk to the right.

\section{REFERENCES}

1) Takahashi H, Tanaka T, Kaneko S: Motion capture for 3D human motion measurement by using one camera with polygonal markers. Transactions of the Society of Instrument and Control Engineers, 2006, 42: 719-726.

2) Hatze $\mathrm{H}$ : High-precision three-dimensional photogrammetric calibration and object space reconstruction using a modified DLT-approach. J Biomech, 1988, 21: 533-538.

3) Watari K, Takeda H, Oguro K, et al: Sports disorders due to kendo in young persons. Journal of Japanese Society for Clinical Sports Medicine, 1987, 4 (Suppl): 18-22.

4) Sports Safety Association: A survey of injuries during activities such as sports. Sports Safety Association, 1999, 21-24.

5) Waku K, Kawano I, Nakamura M, et al.: Analysis of sports injuries in Kendo practitioners analyzed in relation to characteristics of Kendo as a sport.
Research Journal of Budo, 1991, 24 (1): 45-51.

6) Kidera E, Oda N: Knowledge about the living body desirable for Kendo practitioners. Tokyo: Taishukan Shoten, 2006, pp90-93.

7) Nakiri F, Asami T, Takahashi A: A study of the standing position of Kendo practitioners. Research Journal of Budo, 1977, 9 (2): 19-21.

8) Baba H, Imamura K, Iwasaki K: A survey of orthopedic sports injury among children practicing Kendo. Japanese Journal of Orthopaedic Sports Medicine, 1997, 15: 86-87.

9) Kishi S, Sakai N, Sumiya H, et al: Evaluation and physical therapy for the lumbago of junior kendo players. Kansai Clinical Sports Medicine and Science, 2001, 11: 21-23.

10) Shirado O, Kaneda K: Clinically useful kinesiology. Lumbar spine kinesiology - Spinal functions. Journal of Clinical Rehabilitation, 1995, 4(7): 653-657 (in Japanese).

11) White AA, Panjabi MM: Clinical biomechanics of the spine, 2nd ed. Philadelphia: Lippincott, 1990, pp99105.

12) White AA, Panjabi MM: The basic kinematics of the human spine. Spine, 1978, 3: 12-20.

13) Yamamoto I: Effects of lumbar spine posture on entire lumbar spine motions. Proceedings of the Annual Meeting of the Japanese Society for Orthopaedic Biomechanics, 1990, 12: 197-200 (in Japanese).

14) Fujii R, Mukai $Y$, Hoshino N, et al: In vivo $3 D$ motion analysis of lumbar spine-Coupling motion associated with lumbar spine rotation. J Japan Spine Research Society, 2005, 16 (1): 763-769 (in Japanese).

15) Mimura M: Three-dimensional measurement of bone arrangement (7-Three-dimensional analysis of lumbar spine rotation. Spine Spinal Cord, 1995, 8 (8): 637641 (in Japanese). 